\title{
Multiplexed bioluminescence imaging of cancer cell response to hypoxia and inflammation in the caudal- artery injection model of bone metastasis during zoledronic acid treatment
}

\author{
Misa Minegishi ${ }^{1}$, Takahiro Kuchimaru ${ }^{1,2}$, Kenji Nakagawa ${ }^{1}$, Tatsuhiro Isozaki ${ }^{1}$, Satoshi Fujimori ${ }^{1}$, Tetsuya \\ Kadonosono', Shinae Kizaka-Kondoh ${ }^{1}$ \\ 'School of Life Science and Technology, Tokyo Institute of Technology, Kanagawa 226-8501, Japan. \\ ${ }^{2}$ Center for Molecular Medicine, Jichi Medical University, Tochigi 329-0498, Japan. \\ Correspondence to: Prof. Shinae Kizaka-Kondoh, School of Life Science and Technology, Tokyo Institute of Technology, 4259 \\ Nagatsuta, Midori-ku, Yokohama, Kanagawa 226-8501, Japan. E-mail: skondoh@bio.titech.ac.jp \\ How to cite this article: Minegishi M, Kuchimaru T, Nakagawa K, Isozaki T, Fujimori S, Kadonosono T, Kizaka-Kondoh S. \\ Multiplexed bioluminescence imaging of cancer cell response to hypoxia and inflammation in the caudal-artery injection model \\ of bone metastasis during zoledronic acid treatment. J Cancer Metastasis Treat 2021;7:5. \\ http://dx.doi.org/10.20517/2394-4722.2020.96
}

Received: 3 Sep 2020 First Decision: 4 Nov 2020 Revised: 16 Nov 2020 Accepted: 29 Dec 2020 Published: 13 Jan 2021

Academic Editors: Ian Judson, Bal L. Lokeshwar, Sanjay Gupta Copy Editor: Monica Wang Production Editor: Jing Yu

\begin{abstract}
Aim: Therapeutic agents suppressing bone remodeling have been clinically approved to delay metastatic progression and skeletal-related events in patients with bone metastasis. However, therapeutic agents including zoledronic acid (ZA) are insufficient to regress established bone metastasis. Therefore, new treatment strategies are desired, and unraveling the status of cancer cells during bone metastatic progression will help develop therapeutic strategies.
\end{abstract}

Methods: We developed a unique multiplexed reporter system for bioluminescent imaging (MRS-BLI) using three luciferase reporter genes. This system allows for the noninvasive and quantitative monitoring of tumor growth and activities of nuclear factor-kappa B (NF- $\mathrm{B}$ ) and hypoxia-inducible factor (HIF), which are the key transcriptional factors in response to inflammation and hypoxia, respectively. PC-3/MRS-BLI, a human prostate cancer cell line that stably retains the MRS-BLI reporter genes, was applied to the caudal-artery injection model of bone metastasis to observe the status of cancer cells during bone metastasis development and ZA treatment ( $<1$ month).

Results: MRS-BLI reveals key events during the bone metastasis development: NF- $\mathrm{KB}$ and HIF are activated in cancer cells after migration to the bone marrow and are transiently reduced, followed by rapid activation before

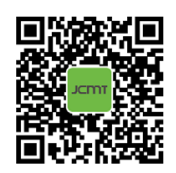


proliferation begins. ZA treatment suppresses the growth of metastasized cancer cells by suppressing NF-kB and HIF activities that may be indirectly induced by osteoclast activation.

Conclusion: By visualizing the NF- $\mathrm{KB}$ and HIF activities of PC-3/MRS-BLI in bone, MRS-BLI has enabled new discoveries regarding the regulation of bone metastases. Further analysis of the progression of bone metastases using MRS-BLI may provide important information for developing new therapeutic strategies.

Keywords: Bone metastasis, multiplexed bioluminescence imaging, zoledronic acid, nuclear factor-kappa B, hypoxia inducible factor

\section{INTRODUCTION}

The bone is frequently affected with metastases in patients with primary tumors in various tissues including the prostate, breast, lung, and kidney ${ }^{[1]}$. Once bone metastasis is developed, patients suffer from pathogenic fractures, nerve compression and hypercalcemia ${ }^{[2]}$. These events are caused by aberrant bone remodeling through destructive osteolytic and/or bone-forming osteoblastic lesions associated with bone metastasis. Therefore, the management of aberrant bone remodeling is a current primary strategy to improve the quality of life of patients with bone metastasis. To improve treatment strategy, it is necessary to understand the states of cancer cells during the formation and progression of bone metastases, but little research has been done on them. It has been suggested that inflammatory and hypoxic microenvironments contribute to bone metastasis development ${ }^{[3-5]}$. Nuclear factor-kappa $\mathrm{B}(\mathrm{NF}-\kappa \mathrm{B})$ is a transcription factor that activates pro-tumor cellular programs in response to inflammation-related stimulations and plays a crucial role in the growth of bone metastasis ${ }^{[6]}$. Tumor tissues often contain hypoxic microenvironments due to aberrant angiogenesis, and hypoxia-inducible transcription factors (HIFs) are activated in metastasized cancer cells, promoting adaptation and growth in the bone marrow ${ }^{[7-9]}$. In addition to the pathological hypoxia, bone marrow is physiologically hypoxic ${ }^{[10]}$. The pathological and physiological hypoxic microenvironments might be involved in the progression of bone metastasis.

Zoledronic acid (ZA), a third-generation aminobisphosphonate, is the gold standard as well as denosumab for the medical management of skeletal relevant events such as osteoclast activities and bone destruction in bone metastasis ${ }^{[11]}$. ZA treatment significantly decreased the incidence of skeletal relevant events in patients with osteolytic bone lesions ${ }^{[12]}$. In addition, ZA is recommended as an adjuvant therapy in breast cancers to prevent bone metastases, most of which are osteolytic ${ }^{[13]}$. ZA further displays efficacy in early treatment of patients with prostate cancers, which are dominantly osteoblastic ${ }^{[14]}$. These observations indicate that ZA is therapeutically effective in both osteolytic and osteoblastic bone metastases. However, ZA treatment is insufficient to regress established bone metastasis and improve the overall survival rate of the patients ${ }^{[15,16]}$. Therefore, ZA combination treatment strategies are widely used clinically ${ }^{[17]}$. Understanding the status of cancer cells in the bone marrow during the development and progression of metastasis will help develop therapeutic strategies. However, there is currently no adequate model to investigate the status of metastasized cells in the bone in real time, and therefore it is difficult to assess the efficacy of drugs in preventing metastatic progression.

Bioluminescence imaging (BLI) is a powerful technique for noninvasive real-time monitoring of various cellular states such as cell proliferation and transcriptional activities in small animals ${ }^{[18]}$. Therefore, a variety of natural and artificial luciferases, and their specific substrates, are used in studies to explore the biological mechanisms, disease development and therapeutic strategies ${ }^{[19]}$. Firefly luciferase (Fluc) has been primarily employed as a reporter gene, and other luciferases such as Renilla luciferase (Rluc), Vargula luciferase (Vluc), and Cypridina luciferase (Cluc) are used in combination with Fluc because of no cross-reactivity 
of luciferin-luciferase reactions ${ }^{[20,21]}$. Previous studies described dual-luciferase reporter systems, which monitors transcription growth factor-beta (TGF- $\beta$ ) induced Smad transcriptional activities and metastatic cancer cell proliferation in murine bone metastatic lesions by Fluc and Rluc reporter genes ${ }^{[2,23]}$. Further multiplexing of BLI would delineate informative cellular states of cancer cells during metastatic progression and may provide information to facilitate new therapeutic strategies to prevent the development and progression of bone metastasis.

Here, we developed a unique multiplexed reporter system for bioluminescent imaging (MRS-BLI) by using three luciferase reporter genes, which allows for the noninvasive and quantitative monitoring of tumor growth as well as NF- $\mathrm{BB}$ and HIF activities. In this work, the stable transfectant of human prostate cancer cell PC-3 of MRS-BLI (PC-3/MRS-BLI) was analyzed shortly after injection with our original caudal-artery injection model of bone metastasis, which allowed us to efficiently deliver PC-3/MRS-BLI into the bone marrow without the need for high technical proficiency ${ }^{[24]}$. We demonstrated the noninvasive monitoring of MRS-BLI signals emanating from bone metastasis in mice hind limbs shortly after injection of PC-3/ MRS-BLI. In addition, MRS-BLI revealed that ZA treatment suppressed NF- $\kappa \mathrm{B}$ and HIF activities as well as the growth of bone metastasis. Thus, MRS-BLI successfully visualized the activities of NF- $\kappa \mathrm{B}$ and HIF in PC-3/MRS-BLI in bones. Taken together, these findings suggest that MRS-BLI could be a powerful tool to develop new therapeutic strategies for bone metastasis.

\section{METHODS}

\section{Reagents}

For cell cultures, we used Roswell Park Memorial Institute (RPMI)-1640 (Nacalai tesque, Kyoto, Japan) and Dulbecco's Modified Eagle Medium (DMEM) (Nacalai tesque, Kyoto, Japan), supplemented with fetal bovine serum (FBS) (Gibco, NY, USA), penicillin and streptomycin (Nacalai tesque, Kyoto, Japan). As selection reagents for cell cloning, we used hygromycin B (Wako, Tokyo, Japan), blasticidin S (Life Technologies, CA, USA), and G418 (Wako, Tokyo, Japan). Zoledronic acid monohydrate (Sigma-Aldrich, MO, USA) was used for in vitro and in vivo studies. The luciferases and substrates used in this study are summarized in Table $1^{[21,25-27]}$.

\section{Plasmid Construction}

We amplified Cluc cDNA with pCL-sv (ATTO, Tokyo, Japan) and then inserted it into the EcoRV site of pcDNA3.1/myc-HisA (Thermo Fisher Scientific, MA, USA) to obtain pcDNA3.1/CMV-Cluc. To construct the pcDNA3.1/CMV-Cluc-glycosylphosphatidylinositol (GPI) plasmid, the EGFP coding sequence of pCAG-EGFP-GPI ${ }^{[28]}$ was replaced with the Cluc coding sequence, and then the Cluc-GPI fragment was amplified and inserted into the EcoRV site of pcDNA3.1/myc-HisA. We obtained pGL4.32/5HRE-CMVmp-

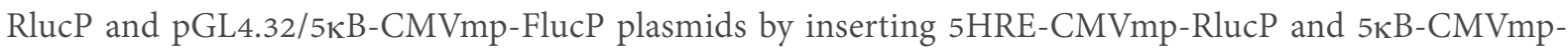
FlucP between the KpnI and EcoRI recognition sites of pGL4.32 (Promega, WI, USA), respectively ${ }^{[2]}$.

\section{Cells}

The human prostate cancer cell lines, PC-3 (ATCC, VA, USA) and PC-3/MRS-BLI, were cultured in an RPMI-1640 medium supplemented with penicillin (100 unit $/ \mathrm{mL}) /$ streptomycin $(100 \mu \mathrm{g} / \mathrm{mL})$ and $10 \% \mathrm{FBS}$. The human cervical cancer HeLa cells (ATCC, VA, USA) were cultured in a DMEM medium supplemented with penicillin $(100 \mathrm{unit} / \mathrm{mL}) /$ streptomycin $(100 \mu \mathrm{g} / \mathrm{mL})$ and $10 \% \mathrm{FBS}$. The cells were maintained at $37^{\circ} \mathrm{C}$, with $5 \% \mathrm{CO}_{2}$, and regularly checked for mycoplasma contamination by a mycoplasma check kit.

\section{Cluc activity measurement}

PC-3 $\left(5.0 \times 10^{4}\right)$ cells were seeded onto 24 -well plates with $1 \mathrm{~mL}$ medium and cultured overnight. The cells were transfected with pcDNA3.1/CMV-Cluc or pcDNA3.1/CMV-Cluc-GPI by polyethylenimine. After $24 \mathrm{~h}$, the cultured medium was harvested and the cells were lysed with a $100 \mu \mathrm{L}$ passive lysis buffer (Promega, 
Table 1. Luciferases used in MRS-BLI

\begin{tabular}{llcclcc}
\hline Luciferase & \multicolumn{1}{c}{ Species } & Molecular weight & Emission wavelength & \multicolumn{1}{c}{ Substrate } & Co-factor & Ref. \\
\hline Firefly & Photinus pyralis & $62 \mathrm{kDa}$ & $565 \mathrm{~nm}$ & $\begin{array}{l}\text { D-luciferin } \\
\text { (PerkinElmer, MA, USA) }\end{array}$ & $\begin{array}{c}\text { ATP } \\
\mathrm{O}_{2}\end{array}$ & {$[25,26]$} \\
Renilla & Renilla reniformis & $36 \mathrm{kDa}$ & $480 \mathrm{~nm}$ & $\begin{array}{l}\text { coelenterazine } \\
\text { (Wako, Tokyo, Japan) }\end{array}$ & $\mathrm{O}_{2}$ & {$[27]$} \\
Cypridina & Cypridina noctiluca & $61 \mathrm{kDa}$ & $465 \mathrm{~nm}$ & $\begin{array}{l}\text { vargulin } \\
\text { (Nanolight technology, AZ, USA) }\end{array}$ & $\mathrm{O}_{2}$ & {$[21]$} \\
\hline
\end{tabular}

MRS-BLI: multiplexed reporter system for bioluminescent imaging

WI, USA). The medium and lysed cells were centrifuged, and Cluc activity in $10 \mu \mathrm{L}$ of the supernatant was measured after mixing with $10 \mu \mathrm{L}$ of vargulin $(0.4 \mu \mathrm{g} / \mathrm{mL})$ using GL-210 luminometer (Microtech Nichion, Chiba, Japan). To measure the correlation between Cluc activity and cell number, PC-3/MRS-BLI were harvested with Accutase (Innovative Cell Technologies, Inc., CA, USA). Cell numbers of $1.25 \times 10^{4}, 2.5 \times$ $10^{4}, 5.0 \times 10^{4}$, and $1.0 \times 10^{5}$ were lysed with $100 \mu \mathrm{L}$ passive lysis buffer and Cluc activity was measured as described above.

\section{Cross-reactivity measurement of Cluc}

HeLa $\left(5.0 \times 10^{4}\right)$ cells were seeded onto 24 -well plates and cultured overnight. The cells were transfected with pcDNA3.1/CMV-Fluc or pcDNA3.1/CMV-Rluc or pcDNA3.1/CMV-Cluc-GPI by polyethylenimine. After $24 \mathrm{~h}$, the cells were lysed with $100 \mu \mathrm{L}$ passive lysis buffer and $10 \mu \mathrm{L}$ of the centrifuged lysate was measured for luciferase activity after mixing with $10 \mu \mathrm{L}$ of the following substrates: coelenterazine-h $(10 \mu \mathrm{g} / \mathrm{mL})$ for 5 HRE-CMVmp-RlucP; luciferase assay reagent for $5 \kappa \mathrm{B}-\mathrm{CMVmp}$-FlucP; vargulin $(0.4 \mu \mathrm{g} / \mathrm{mL})$ for CMV-Cluc-GPI.

\section{Establishment of PC-3/MRS-BLI}

PC-3 cells were transfected with pGL4.32/5HRE-CMVmp-RlucP plasmid by the calcium phosphate method and cultured in selection medium containing $200 \mu \mathrm{g} / \mathrm{mL}$ hygromycin B. To establish PC-3/5HRE-CMVmpRlucP, colonies were selected by measuring the bioluminescence (BL) of multiple colonies cultured in $1 \%$ $\mathrm{O}_{2}$. PC-3/5HRE-CMVmp-RlucP were co-transfected with pGL4.32/5кB-CMVmp-FlucP plasmid and pEF6/ Myc-HisA (Thermo Fisher Scientific, MA, USA), and were selected in a selection medium containing $6 \mu \mathrm{g} / \mathrm{mL}$ blasticidin S. To establish PC-3/5HRE-CMVmp-RlucP, $5 \kappa \mathrm{B}$-CMVmp-FlucP, we assessed the BL of multiple colonies cultured with $5 \mathrm{ng} / \mathrm{mL}$ hTNF- $\alpha$ (Rocky Hill, NJ, USA). We then transfected PC-3/5HRE-CMVmpRlucP, $5 \kappa$ B-CMVmp-FlucP with pcDNA3.1/CMV-Cluc-GPI by ScreenFect ${ }^{\mathrm{TM}} \mathrm{A}$ (Wako, Tokyo, Japan), followed by selection with $500 \mu \mathrm{g} / \mathrm{mL}$ G418. We assessed the BL of multiple antibiotic-resistant colonies to establish PC-3/MRS-BLI.

\section{Mice}

SCID mice (male) were obtained from Charles River Laboratories (Kanagawa, Japan). All mice were provided access to food and water ad libitum and were housed in animal facilities at the Tokyo Institute of Technology. All experimental procedures using mice were approved by the Animal Experiment Committee of the Tokyo Institute of Technology (authorization number 201006-3), and carried out in accordance with relevant national and international guidelines.

\section{Bone metastasis model with ZA treatment}

PC-3/MRS-BLI $\left(1.0 \times 10^{6}\right.$ cells $)$ was suspended in $100 \mu \mathrm{L}$ PBS and was injected into the caudal artery of SCID mice under anesthesia conditions ${ }^{[24]}$. A stock solution of ZA was prepared by dissolving ZA monohydrate (Sigma-Aldrich, MO, USA) in sterile $\mathrm{ddH}_{2} \mathrm{O}$ at a concentration of $10 \mathrm{mM}$, divided into small aliquots, and kept at $-80^{\circ} \mathrm{C}$ for long-term storage. Mice were intravenously administered with $100 \mu \mathrm{L}$ of either $100 \mu \mathrm{g} / \mathrm{mL}$ ZA or PBS at 5-day intervals from day 2 to day 22 . 


\section{In vivo MRS-BLI}

All bioluminescence image data of tumor bearing mice were acquired with IVIS Spectrum (PerkinElmer, MA, USA). 5HRE-CMVmp-RlucP images were acquired at 2 min after intravenous injection of coelenterazine-h $(250 \mu \mathrm{g} / \mathrm{kg}) .2 \mathrm{~h}$ later, CMV-Cluc-GPI images were acquired at $30 \mathrm{sec}$ after intravenous injection of vargulin $(2 \mu \mathrm{g} / \mathrm{kg})$. After another $2 \mathrm{~h}, 5 \kappa \mathrm{B}-\mathrm{CMVmp}$-FucP images were acquired at $15 \mathrm{~min}$ after intraperitoneal injection of D-luciferin $(50 \mathrm{mg} / \mathrm{kg})$. For each imaging session, mice are immobilized by gas anesthesia mixing $2 \%$ isoflurane and $98 \%$ oxygen. The following conditions were used for image acquisition: open emission filter, exposure time $=0.5 \mathrm{~min}$ for $5 \mathrm{HRE}-\mathrm{CMVmp}-\mathrm{RlucP}$ and CMVCluc-GPI, exposure time $=1 \mathrm{~min}$ for $5 \mathrm{\kappa B}$-CMVmp-FucP, binning $=$ medium $: 8$, field of view $=13.4 \mathrm{~cm}$ $\times 13.4 \mathrm{~cm}$, and $\mathrm{f} / \mathrm{stop}=1$. The BL images were analyzed by Living Image 4.3 software (PerkinElmer, MA, USA) that is specialized for IVIS.

\section{PC-3/MRS-BLI proliferation assay with ZA treatment}

PC-3/MRS-BLI $\left(4.0 \times 10^{3}\right)$ cells were seeded onto 96-well plates, and cultured overnight. The cells were further cultured with ZA $(0.05,0.5,5,50,100 \mu \mathrm{M})$ for 0,24 or $48 \mathrm{~h}$. After the culture, cell proliferation rates were assessed with WST-1 reagents (Roche Applied Science, Penzberg, Germany) according to the manufacturer's protocol.

\section{MRS-BLI reporter assay with ZA treatment}

PC-3/MRS-BLI $\left(5.0 \times 10^{4}\right)$ cells were seeded onto 24 -well plates, and cultured overnight. The cells were further cultured with ZA $(0.05,0.5,5 \mu \mathrm{M})$ for $16 \mathrm{~h}$. The cells were lysed with a $100 \mu \mathrm{L}$ passive lysis buffer (Promega, WI, USA) and centrifuged. Luciferase activity of $10 \mu \mathrm{L}$ supernatant was measured using the GL210 luminometer (Microtech Nichion, Chiba, Japan) after mixing with $10 \mu \mathrm{L}$ of the following substrates: coelenterazine-h $(10 \mu \mathrm{g} / \mathrm{mL})$ for $5 \mathrm{HRE}$-CMVmp-RlucP; luciferase assay reagent for $5 \kappa \mathrm{B}$-CMVmp-FlucP; vargulin $(0.4 \mu \mathrm{g} / \mathrm{mL})$ for CMV-Cluc-GPI.

\section{Statistical analysis}

Data are presented as means \pm standard error of the mean (SEM) and were statistically analyzed with a two-side Student's $t$-test. $P$-values $<0.05$ were considered statistically significant.

\section{RESULTS}

Herein, we have constructed a new bioluminescence reporter, Cluc-GPI, for use in combination with Fluc and Rluc reporters that are commonly employed in bioluminescence imaging. In reaction with vargulin, Cluc generates a stronger bioluminescence than Vluc, which was isolated from marine ostracods as Cluc was $^{[21]}$. However, wild-type Cluc is naturally secreted from cells and is stable in blood circulation, thus increasing background signals in mice ${ }^{[30]}$. Therefore, the GPI signal sequence of CD90 was added to the C-terminus of Cluc so that the created Cluc-GPI was anchored to the cell membrane [Figure $1 \mathrm{~A}]^{[28]}$. As we expected, secretion of Cluc-GPI into the medium was decreased 10-fold compared to wild-type Cluc, and intracellular Cluc activity was significantly increased [Figure 1B]. We further confirmed that Cluc-GPI showed no cross-reactivity with Fluc and Rluc BL systems [Figure 1C]. Overall, our results indicate that Cluc-GPI is a highly sensitive BLI reporter gene and can be used in combination with the Fluc and Rluc dual BL reporters.

We next established the cell line PC-3/MRS-BLI, which stably carries the multiplexed luciferase reporter system. PC-3 is a human prostate cancer cell line, often used in studies of bone metastases in small animal $\operatorname{models}^{[31]}$. To establish PC-3/MRS-BLI, we transduced three reporter genes, CMV-Cluc-GPI (Cluc-GPI), $5 \kappa \mathrm{B}-\mathrm{CMVmp}$-FlucP ( $\mathrm{KB}$-FlucP) and 5HRE-CMVmp-RlucP (HRE-RlucP) [Figure 2A]. Fluc and Rluc were fused with a PEST sequence to shorten the half-life of luciferases, thereby improving the real-time monitoring effect of reporters ${ }^{[32]}$. The $\kappa \mathrm{B}$-FlucP responded as expected to inflammatory cytokine TNF- $\alpha$ in 
A
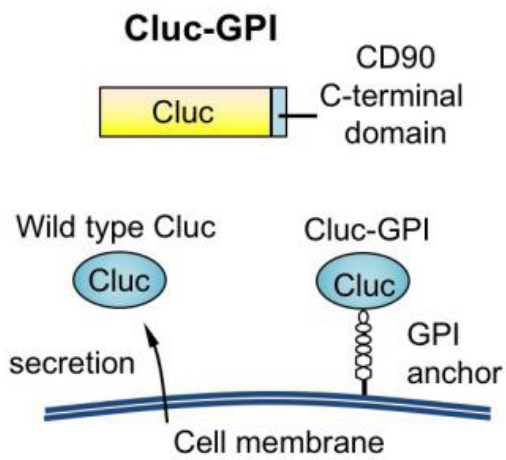

B

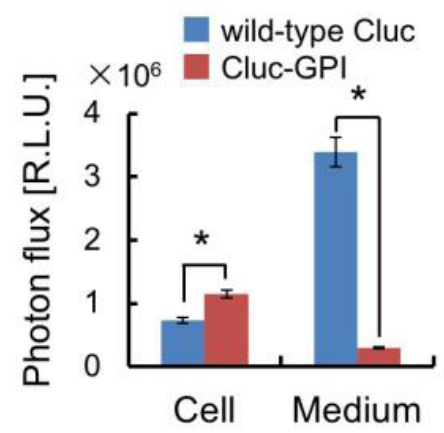

C
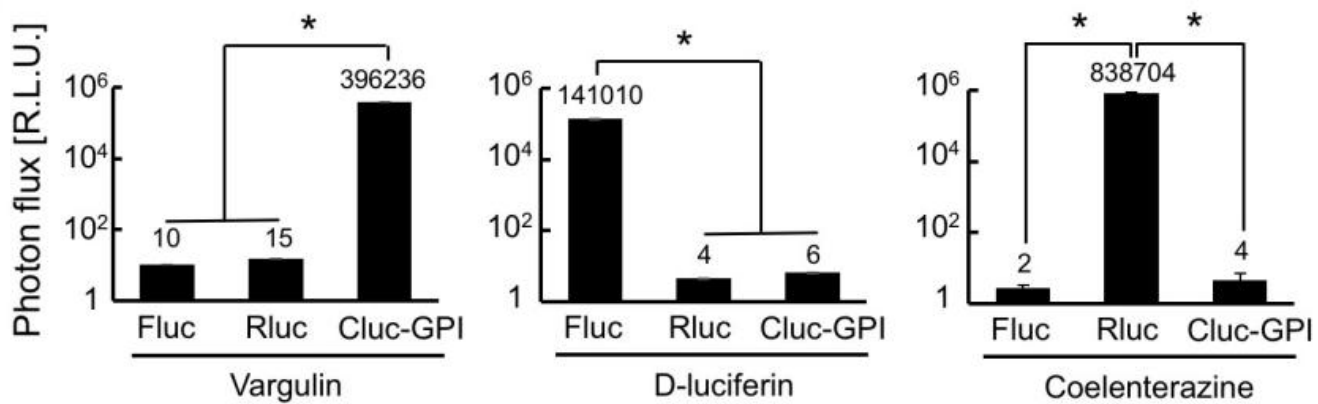

Figure 1. Construction of the Cluc-GPI reporter. A: Schematic of Cluc-GPI. Cluc is fused with the C-terminal domain of CD90; B: Intracellular (Cell) and extracellular (Medium) Cluc bioluminescence intensities were measured $24 \mathrm{~h}$ after transfection of the reporter gene. $n=3,{ }^{\star} P<0.05$; C: Cell lysates of HeLa transfected with Fluc, Rluc or Cluc-GPI reporter genes were measured for bioluminescence intensities after reactions with the indicated luciferase substrates. $n=3,{ }^{\star} P<0.05$. Cluc: Cypridina luciferase; Fluc: Firefly luciferase; Rluc: Renilla luciferase; GPI: glycosylphosphatidylinositol

a dose-dependent manner [Figure 2B]; TNF- $\alpha$ stimulation increased Fluc activity to reach peak levels in $12 \mathrm{~h}$ [Figure 2C]; HRE-RlucP responded to hypoxia when $\mathrm{O}_{2}$ concentration was less than approximately $5 \%$ [Figure 2D]; while Rluc activity peaked at around $18 \mathrm{~h}$ under $1 \% \mathrm{O}_{2}$ conditions [Figure 2E]. Furthermore, stable expression of CMV-Cluc-GPI in PC-3 cells displayed a linear correlation between luminescence intensity and cell number [Figure $2 \mathrm{~F}$ ]. These results indicate that cellular responses to inflammation and hypoxia can be monitored by the BL of Fluc and Rluc, respectively, in addition to monitoring the cell number by Cluc BL.

PC-3/MRS-BLI cells injected through caudal artery were efficiently delivered to the hindlimb bone and developed metastases as reported previously ${ }^{[24]}$. Treatment with ZA $(0.4 \mathrm{mg} / \mathrm{kg})$ was started 2 days after the caudal artery injection of PC-3/MRS-BLI cells, and ZA was intravenously injected 5 times at 5-day intervals [Figure $3 \mathrm{~A}$ ]. BLI monitoring NF- $\mathrm{KB}$ and HIF activities and tumor mass were sequentially acquired at 2-hour intervals to minimize the influence of time lag and residual substrates [Figure 3A]. The MRS-BLI was noninvasively observed from day 1 . PC-3/MRS-BLI cell growth continued over time and significantly accelerated on day 17 [Figure $3 \mathrm{~B}$ and C]. ZA treatment significantly suppressed PC-3/MRS-BLI cell growth [Figure $3 \mathrm{C}$ ]. Changes in NF- $\kappa \mathrm{B}$ and HIF activities per cancer cell were quantitatively analyzed by normalizing the BL intensities of Fluc and Rluc by that of Cluc (tumor mass). NF- $\kappa \mathrm{B}$ and HIF increased rapidly prior to tumor growth [Figure $3 \mathrm{~B}, \mathrm{D}$ and $\mathrm{E}$ ]. Interestingly, ZA treatment significantly suppressed transcriptional activities by day 12, which was earlier than cell growth suppression, namely, by day 17 [Figure $3 \mathrm{C}-\mathrm{E}$ ]. These results suggested that ZA treatment suppresses the growth of metastasized cells by reducing microenvironmental inflammation and hypoxia. 
A

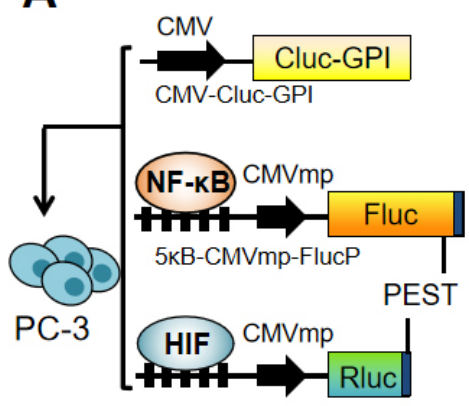

5HRE-CMVmp-RlucP

D

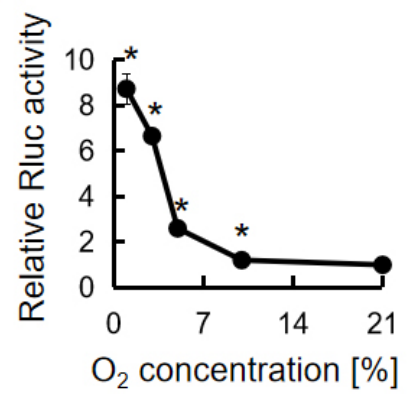

B

E

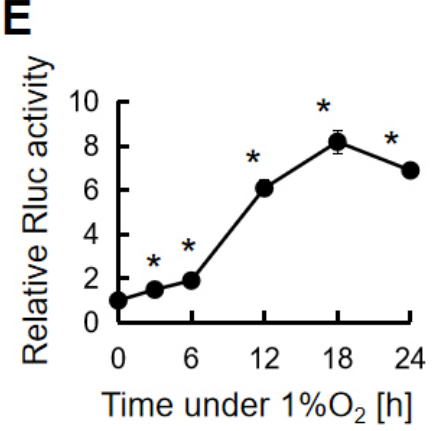

C

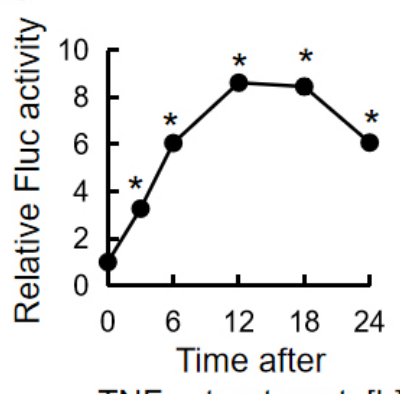

TNF- $\alpha$ treatment $[\mathrm{h}]$

$\mathbf{F}$

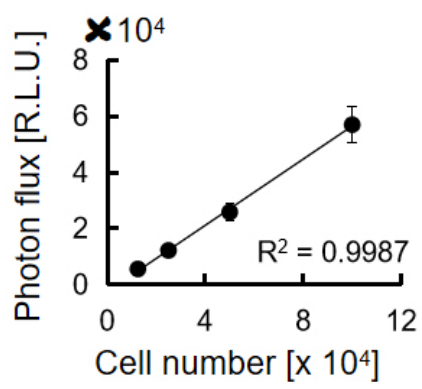

Figure 2. Establishment of human prostate cancer, $P C-3 / M R S-B L I$, stably carrying multiplexed luciferase reporters. A: Schematic of PC-3/MRS-BLI. The $5 \kappa B$ motif contains five tandem repeats of the GGACTTTCC. The 5HRE motif contains five tandem repeats of the ACGTGG; B: Relative $\kappa B-F l u c P$ bioluminescence intensity in PC-3/MRS-BLI cells treated with TNF- $\alpha(0.01,0.1,0.1,5 \mathrm{ng} / \mathrm{mL})$ for 6 h. $n=3,{ }^{*} P<0.05$ to the non-treated cells; C: Time-course changes of $\kappa B$-FlucP bioluminescence intensity in TNF- $\alpha$ ( $5 \mathrm{ng} /$ $\mathrm{mL}$ ) treated PC-3/MRS-BLI cells. $n=3,{ }^{\star} P<0.05$ to $0 \mathrm{~h}$; D: Relative HRE-RlucP bioluminescence intensity in PC-3/MRS-BLI cells at various $\mathrm{O}_{2}$ concentrations $(1,3,5,10,21 \%)$ for $16 \mathrm{~h} . n=3,{ }^{\star} P<0.05$ to the cell under $21 \% \mathrm{O}_{2}$; E: Time-course changes of HRE-RlucP bioluminescence intensity in PC-3/MRS-BLI cells under $1 \% \mathrm{O}_{2} \cdot n=3,{ }^{\star} P<0.05$ to $0 \mathrm{~h}$; F: Correlation of PC-3/MRS-BLI cell number and Cluc BL intensity. $n=3$ for each cell number. MRS-BLI: multiplexed reporter system for bioluminescent imaging; Cluc: Cypridina luciferase; Fluc: Firefly luciferase; Rluc: Renilla luciferase; GPI: glycosylphosphatidylinositol; TNF- $\alpha$ : tumor necrosis factor-alpha; NF- $\mathrm{B}$ : nuclear factor-kappa B; HIF: hypoxia-inducible factor

To determine whether these in vivo BLI results reflected the direct effects of ZA on PC-3/MRS-BLI proliferation and $\mathrm{NF}_{-} \mathrm{KB}$ and HIF activities, in vitro assays were performed. First, in vitro assessment of the ZA effect on PC-3/MRS-BLI cell proliferation reveals that ZA treatment, for up to $24 \mathrm{~h}$, did not influence PC-3/MRS-BLI cell proliferation even at the highest concentration of $100 \mu \mathrm{M}$ [Figure $4 \mathrm{~A}$ ]. Prolonged ZA treatment $(48 \mathrm{~h})$ showed significant suppression at higher concentrations $(50$ and $100 \mu \mathrm{M})$ [Figure $4 \mathrm{~A}$ ]. These concentrations are much higher than those used in the in vivo treatment (data shown in Figure 3), in which the calculated ZA concentration in metastatic lesions is less than $5 \mu \mathrm{M}$. Cellular BL assays of PC-3/MRS-BLI cells with D-luciferin and coelenterazine revealed that Fluc and Rluc activities corresponding to NF- $\mathrm{B}$ and HIF transcriptional activities, respectively, were unaffected by ZA treatment at less than $5 \mu \mathrm{M}$ [Figure $4 \mathrm{~B}$ ]. Overall, the results suggested that the observed in vivo effects of ZA on PC-3/ MRS-BLI might be secondary effects: ZA reduced inflammation and hypoxia in the microenvironment surrounding PC-3/MRS-BLI, probably through its inhibitory effect on bone destruction [Figure 4C].

\section{DISCUSSION}

In this study, we developed a new multiplexed BLI system, and visualized proliferation and activities of key transcriptional factors of cancer cells, NF- $\kappa \mathrm{B}$ and HIF, during metastasis development and ZA treatment in bone. Of the three reporters used here, we found the newly constructed Cluc-GPI reporter useful: its strong bioluminescence overcomes the poor tissue-penetration of visible wavelength light of Cluc ( $\lambda$ max $=465 \mathrm{~nm}$ ), allowing the growth of metastasized cancer cells in the mice hind limbs to be monitored from 
A

\section{ZA administration}

$$
\begin{aligned}
& \text { ZA }(0.4 \mathrm{mg} / \mathrm{kg} \text {, i.v.) or PBS } \\
& \begin{array}{lllllll}
0 & 2 & 7 & 12 & 17 & 22 & 27
\end{array}
\end{aligned}
$$

Days after PC-3/MRS-BLI injection

\section{Substrate administration}

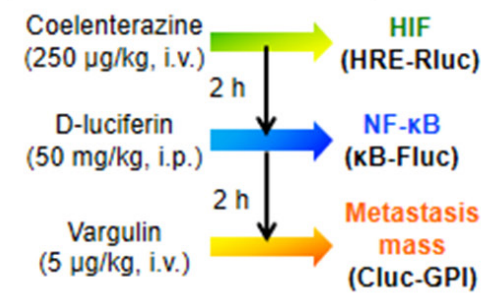

C

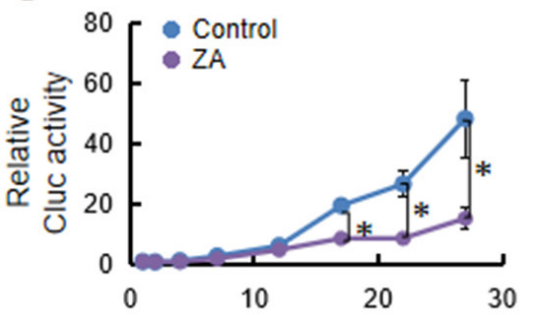

Days after injection of PC-3/MRS-BLI

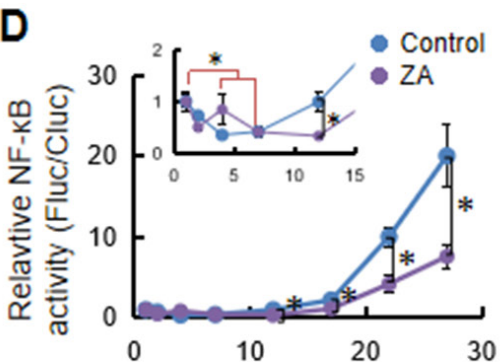

Days after injection of PC-3/MRS-BLI
B

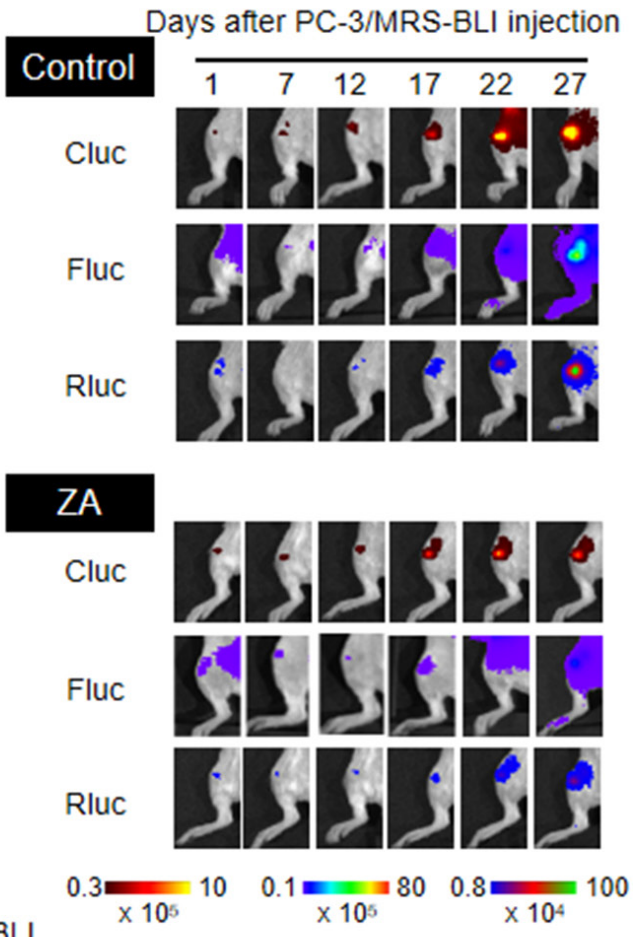

E

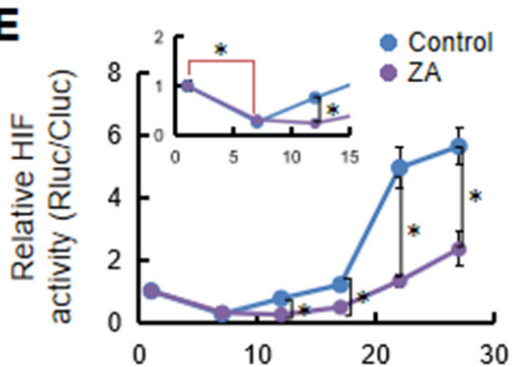

Days after injection of PC-3/MRS-BLI

Figure 3. Non-invasive observation of bone metastasis of PC-3/MRS-BLI. A: Schematic demonstrating the administration of ZA or substrates for MRS-BLI in a murine bone metastasis model with PC-3/MRS-BLI; B: Multiplexed bioluminescent imaging of bone metastasis of PC-3/MRS-BLI treated with or without ZA; C: Relative metastasis mass of PC-3/MRS-BLI bone metastasis monitored with CMV-Cluc-GPI. $n=7$ (Control), $n=5$ (ZA), ${ }^{*} P<0.05$; D: Relative NF- $\kappa B$ activity ( $\kappa B$-FlucP/CMV-Cluc-GPI) of PC-3/MRSBLI bone metastasis. The inset graph highlights NF- $\kappa B$ activity on days 1 through day $12 . n=7$ (Control), $n=5$ (ZA), ${ }^{\star} P<0.05$. Red lines indicate the statistical significance of day 1 over day 4 and day 7 in the control group; E: Relative HIF activity (HRE-RlucP/CMVCluc-GPI) of PC-3/MRS-BLI bone metastasis. The inset graph highlights HIF activity on day 1 through day $12 . n=7$ (Control), $n=5$ $(Z A),{ }^{\star} P<0.05$. Red lines indicate the statistical significance between day 1 (control group) and day 7. ZA: Zoledronic acid; MRS-BLI: multiplexed reporter system for bioluminescent imaging; Cluc: Cypridina luciferase; Fluc: Firefly luciferase; Rluc: Renilla luciferase; GPI: glycosylphosphatidylinositol; NF-kB: nuclear factor-kappa B; HIF: hypoxia-inducible factor

day 1 of caudal artery injection [Figure 3B]. This suggests that Cluc-GPI may be a new option to combine with Fluc and Rluc reporter genes for multiplexed BLI. Multi-modal techniques combining optical imaging and positron emission tomography, magnetic resonance imaging and X-ray computed tomography have been employed, and have provided valuable information of metastatic lesions ${ }^{[33-35]}$. Multiple optical imaging has also provided us a different benefit from multi-modal imaging, in that, multiple pieces of information can be easily and safely collected from the same individual at almost the same time. MRS-BLI is the first example of applying multiple luciferases to an in vivo imaging system that can visualize mass as well as dual transcriptional activities in bone metastases. 

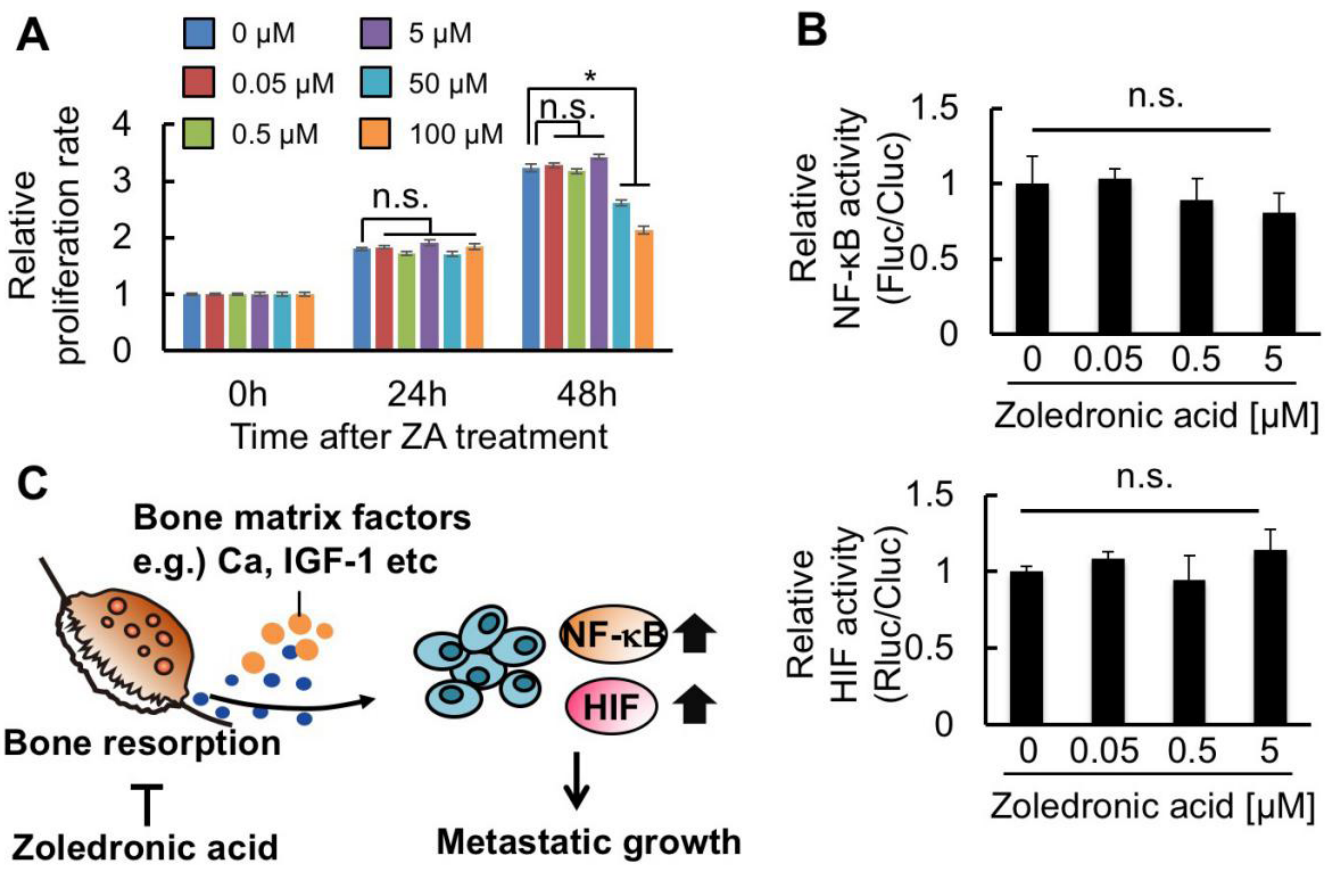

Figure 4. ZA suppresses microenvironmental factors activating NF- $\mathrm{KB}$ and HIF in bone metastatic lesions. A: WST-1 assay of PC-3/ MRS-BLI treated with varied concentrations of ZA. $n=3$, ${ }^{\star} P<0.05$; B: Relative NF- $\kappa B$ (top) and HIF (bottom) activities of PC-3/MRS$\mathrm{BLI}$ treated with varied concentrations of ZA. $n=3$; C: Diagram of the hypothetical mechanism for NF- $\kappa B$ and HIF activation in bonemetastasized cancer cells, and for ZA function suppressing this activation. ZA: Zoledronic acid; NF-אB: nuclear factor-kappa B; HIF: hypoxia-inducible factor; MRS-BLI: multiplexed reporter system for bioluminescent imaging

Despite the significant growth inhibitory effect of ZA on PC3/MRS-BLI cells in vivo [Figure 3C], treatment of cultured PC3/MRS-BLI cells with ZA at concentrations less than $5 \mu \mathrm{M}$ did not show any inhibitory effects on the proliferation [Figure 4A]. ZA treatment at higher concentrations (50 and $100 \mu \mathrm{M})$ resulted in significant growth inhibition of PC3/MRS-BLI cells only for $48 \mathrm{~h}$. This inhibitory effect is consistent with previous studies showing that high concentrations of nitrogen-containing bisphosphonate analogues induce apoptosis in prostate cancer cell lines via inhibition of the mevalonate pathway ${ }^{[3,37]}$. Pharmacokinetic study in rodents has reported a sharp decline in plasma ZA levels: the levels at $4 \mathrm{~h}$ became less than $1 \%$ of plasma levels at $5 \mathrm{~min}$ after intravenous injection ${ }^{[38]}$. This indicates that the ZA concentration in bone metastatic lesions would be predominantly less than $5 \mu \mathrm{M}$. Therefore, the growth inhibitory effects of ZA in vivo are probably due to indirect effects on bone destruction rather than direct effects on cancer cells.

Multiplexed bioluminescent imaging of bone metastasis lesions revealed that NF- $\kappa \mathrm{B}$ and HIF activities per cell were significantly high at day 1 and day 12 , and continued to sharply increase thereafter in control mice [Figure 3D and E]. ZA treatment significantly suppressed this increase [Figure 3D and E]; the inhibitory effects of ZA on NF- $\mathrm{BB}$ and HIF were not direct effects on cancer cells [Figure $4 \mathrm{~B}$ ]. Based on these results, we propose a mechanism for the initiation and progression of bone metastasis: Cancer cells that have migrated to the bone marrow enhance NF- $\mathrm{B}$ and HIF activities in order to adapt to the physiological low partial pressure of oxygen in the bone marrow ${ }^{[10]}$ and respond to immunological attacks on cancer $\operatorname{cells}^{\left[{ }^{[36]}\right.}$, respectively. Cancer cells that are protected by these activities and are able to survive will start growing and activate osteoclasts, which induce bone destruction and release of factors from the bone. The released factors increase NF- $\mathrm{BB}$ and HIF activities in cancer cells [Figure $4 \mathrm{C}$ ]. This model is supported by previous studies that demonstrate that bone-matrix derived insulin growth factors activated NF- $\kappa \mathrm{B}$ and HIF signaling in cancer cells ${ }^{[6,8]}$. In addition, calcium stored in the bone matrix is a potent factor to induce accumulation of $\mathrm{HIF}^{[39]}$. This may be involved in prostate cancer cells, benefiting growth from extracellular calcium in the bone marrow ${ }^{[40]}$. Taken together, bone destruction may trigger activation of NF- $\kappa \mathrm{B}$ and 
HIF signaling in cancer cells, enhancing the growth and malignant progression of cancer cells by inducing various target genes associated with $\mathrm{NF}-\mathrm{\kappa B}$ and HIF.

The multiplexed luciferase reporter system described here would be even more beneficial when combined with a clinically relevant mouse model that can reflect the aging process of the patient's bone marrow. Accumulated evidence suggests that the aging process significantly remodels the microenvironments of mouse and human bones ${ }^{[41,42]}$. Currently, relatively young-aged mice (5-10 weeks) have been used for preclinical studies of bone metastasis. It is unclear whether current murine models adequately reproduce the patient's bone microenvironments. Further studies of the bone microenvironments are thus needed to develop clinically relevant murine models of bone metastasis that accelerate the development of effective therapeutic strategies.

\section{DECLARATIONS}

\section{Acknowledgments}

We are grateful to Shigeaki Watanabe (Summit Pharmaceuticals International Corporation, Tokyo, Japan) for discussion and technical support of IVIS. We also thank Biomaterials Analysis Division, Technical Department of Tokyo Institute of Technology for DNA sequencing analysis.

\section{Authors' contributions}

Designed the overall project: Minegishi M, Kuchimaru T, Kizaka-Kondoh S

Performed the experiments: Minegishi M, Kuchimaru T, Nakagawa K, Isozaki T, Fujimori S

Analyzed and interpreted the data: Minegishi M, Kuchimaru T, Nakagawa K, Kadonosono T, KizakaKondoh S

Wrote the manuscript: Minegishi M, Kuchimaru T, Kizaka-Kondoh S

\section{Availability of data and materials}

The data that support the findings of this study are available from the corresponding author upon request.

\section{Financial support and sponsorship}

This research was supported by a Grant-in-Aid for Scientific Research on Innovative Areas "Integrative Research on Cancer Microenvironment Networks from the Ministry of Education", Culture, Sports, Science and Technology of Japan (S. K-K), Grant-in-Aid for Young Scientist (B) (T. Ku) and Princess Takamatsu Cancer Research Fund (T. Ku).

\section{Conflicts of interest}

The authors declare no competing financial interests.

\section{Ethical approval and consent to participate}

SCID mice (male) were obtained from Charles River Laboratory in Japan (Kanagawa, Japan). All mice were provided access to food and water ad libitum and were housed in the animal facilities at the Tokyo Institute of Technology. All experimental procedures using mice were approved by the Animal Experiment Committee of the Tokyo Institute of Technology (authorization number 201006-3), and carried out in accordance with relevant national and international guidelines.

\section{Consent for publication}

Not applicable.

\section{Copyright}

(c) The Author(s) 2021. 


\section{REFERENCES}

1. Macedo F, Ladeira K, Pinho F, et al. Bone metastases: an overview. Oncol Rev 2017;11:321-8.

2. Tsuzuki S, Park SH, Eber MR, Peters CM, Shiozawa Y. Skeletal complications in cancer patients with bone metastases. Int $J$ Urol 2016;23:825-32.

3. Hanahan D, Weinberg RA. Hallmarks of cancer: the next generation. Cell 2011; 144:646-74.

4. Casimiro S, Guise TA, Chirgwin J. The critical role of the bone microenvironment in cancer metastases. Mol Cell Endocrinol 2009;310:71-81.

5. Suva LJ, Washam C, Nicholas RW, Griffin R. Bone metastasis: mechanisms and therapeutic opportunities. Nat Rev Endocrinol 2011;7:208-18.

6. Hiraga T, Myoui A, Hashimoto N, et al. Bone-derived IGF mediates crosstalk between bone and breast cancer cells in bony metastases. Cancer Res 2012;72:4238-79.

7. Hiraga T, Kizaka-Kondoh S, Hirota K, Hiraoka M, Yoneda T. Hypoxia and hypoxia-inducible factor-1 expression enhance osteolytic bone metastases of breast cancer. Cancer Res 2017;67:4157-63.

8. Kuchimaru T, Hoshino T, Aikawa T, et al. Bone resorption facilitates osteoblastic bone metastatic colonization by cooperation of insulinlike growth factor and hypoxia. Cancer Sci 2014;105:553-9.

9. Johnson RW, Sowder ME, Giaccia AJ. Hypoxia and bone metastatic disease. Curr Osteoporos Rep 2017;15:231-8.

10. Spencer JA, Ferraro F, Roussakis E, et al. Direct measurement of local oxygen concentration in the bone marrow of live animals. Nature 2014;508:269-73.

11. Reszka AA, Rodan GA. Nitrogen-containing bisphosphonate mechanism of action. Mini-Reviews Med Chem 2004;4:711-9.

12. Kohno N, Aogi K, Minami H, et al. Zoledronic acid significantly reduces skeletal complications compared with placebo in Japanese women with bone metastases from breast cancer: a randomized, placebo-controlled trial. J Clin Oncol 2005;23:3314-21.

13. Dhesy-Thind S, Fletcher GG, Blanchette PS, et al. Use of adjuvant bisphosphonates and other bone-modifying agents in breast cancer: a cancer care ontario and american society of clinical oncology clinical practice guideline. J Clin Oncol 2017;35:2062-81.

14. Saad F, Gleason DM, Murray R, et al. Long-term efficacy of zoledronic acid for the prevention of skeletal complications in patients with metastatic hormone-refractory prostate cancer. $J$ Natl Cancer Inst 2004;96: 879-82.

15. Saad F, Gleason DM, Murray R, et al. A randomized, placebo-controlled trial of zoledronic acid in patients with hormone-refractory metastatic prostate carcinoma. J Natl Cancer Inst 2002;94:1458-68.

16. Rosen LS, Gordon D, Tchekmedyian S, et al. Zoledronic acid versus placebo in the treatment of skeletal metastases in patients with lung cancer and other solid tumors: a phase III, double-blind, randomized trial - the zoledronic acid lung cancer and other solid tumors study group. J Clin Oncol 2003;21:3150-7.

17. Gdowski AS, Ranjan A, Vishwanatha JK. Current concepts in bone metastasis, contemporary therapeutic strategies and ongoing clinical trials. J Exp Clin Cancer Res 2017;36:108.

18. Kelkar M, De A. Bioluminescence based in vivo screening technologies. Curr Opin Pharmacol 2012;12:592-600.

19. Yao Z, Zhang BS, Prescher JA. Advances in bioluminescence imaging: new probes from old recipes. Curr Opin Chem Biol 2018;45:148-56.

20. Paley MA, Prescher JA. Bioluminescence: a versatile technique for imaging cellular and molecular features. Physiol Behav 2019;176:139-48.

21. Nakajima Y, Kobayashi K, Yamagishi K, Enomoto T, Ohmiya Y. cDNA cloning and characterization of a secreted luciferase from the luminous Japanese ostracod, Cypridina noctiluca. Biosci Biotechnol Biochem 2004;68:565-70.

22. Katsuno Y, Hanyu A, Kanda H, et al. Bone morphogenetic protein signaling enhances invasion and bone metastasis of breast cancer cells through Smad pathway. Oncogene 2008;27:6322-33.

23. Korpal M, Yan J, Lu X, Xu SW, Lerit DA, Kang YB. Imaging transforming growth factor-B signaling dynamics and therapeutic response in breast cancer bone metastasis. Nat Med 2009;15:960-6.

24. Kuchimaru T, Kataoka N, Nakagawa K, et al. A reliable murine model of bone metastasis by injecting cancer cells through caudal arteries. Nat Commun 2018;9:2981.

25. de Wet JR, Wood KV, DeLuca M, Helinski DR, Subramani S. Firefly luciferase gene: structure and expression in mammalian cells. Mol Cell Biol 1987;7:725-37.

26. Ando Y, Niwa K, Yamada N, et al. Firefly bioluminescence quantum yield and colour change by pH-sensitive green emission. Nat Photonics 2008;2:44-8.

27. Lorenz WW, McCann RO, Longiaru M, Cormier MJ. Isolation and expression of a cDNA encoding Renilla reniformis luciferase. Proc Natl Acad Sci U S A 1991;88:4438-42.

28. Kondoh G, Gao XH, Nakano Y, et al. Tissue-inherent fate of GPI revealed by GPI-anchored GFP transgenesis. FEBS Lett 1999;458:299303.

29. Fujita K, Tanaka Y, Sho T, et al. Intracellular CO release from composite of ferritin and ruthenium carbonyl complexes. J Am Chem Soc 2014;136:16902-8.

30. Charles JP, Fuchs J, Hefter M, et al. Monitoring the dynamics of clonal tumour evolution in vivo using secreted luciferases. Nat Commun 2014;5:3981.

31. Park SH, Eber MR, Shiozawa Y. Models of prostate cancer bone metastasis. Methods Mol Viol 2019;1914:295-308.

32. Leclerc GM, Boockfor FR, Faught WJ, Frawley LS. Development of a destabilized firefly luciferase enzyme for measurement of gene expression. Biotechniques 2000;29:590-601.

33. Kang YB, he W, Tulley S, et al. Breast cancer bone metastasis mediated by the Smad tumor suppressor pathway. Proc natl Acad Sci U S A 2005;102:13909-14. 
34. Henriquez NV, van Overveld PG, et al. Advances in optical imaging and novel model systems for cancer metastasis research. Clin Exp Metastasis 2007;24:699-705.

35. Brisset JC, Hoff BA, Chenevert TL, et al. Integrated multimodal imaging of dynamic bone-tumor alterations associated with metastatic prostate cancer. PLoS One 2015;10:e123877.

36. Miki T, Yano S, Hanibuchi M, Sone S. Bone metastasis model with multiorgan dissemination of human small-cell lung cancer (SBC-5) cells in natural killer cell-depleted SCID Mice. Oncol Res 2000;12:209-17.

37. Oades GM, Senaratne SG, Clarke IA, Kirby RS, Colston KW. Nitrogen containing bisphosphonates induce apoptosis and inhibit the mevalonate pathway, imparing RAS membrain localization in prostate cancer cells. $J$ Urol 2003;170,246-52.

38. Weiss HM, Pfaar U, Schweitzer A, Wiegand H, Skerjanec A, Schran H. Biodistribution and plasma protein binding of zoledronic acid. Drug Metab Dispos 2008;36:2043-9.

39. Hui AS, Bauer AL, Striet JB, Schnell PO, Czyzyk-Krzeska MF. Calcium signaling stimulates translation of HIF- $\alpha$ during hypoxia. FASEB $J$ 2006;20:466-75.

40. Liao J, Schneider A, Datta NS, McCauley LK. Extracellular calcium as a candidate mediator of prostate cancer skeletal metastasis. Cancer Res 2006;66:9065-73.

41. Burr DB. Changes in bone matrix properties with aging. Bone 2019;120,85-93.

42. Ho YH, Méndez-Ferrer S. Microenvironmental contributions to hematopoietic stem cell aging. Haematologica 2020;105:38-46. 